\title{
Behavioral Analysis of Signals that Guide Learned Changes in the Amplitude and Dynamics of the Vestibulo-Ocular Reflex
}

\author{
Jennifer L. Raymond and Stephen G. Lisberger \\ Department of Physiology, W. M. Keck Foundation Center for Integrative Neuroscience, and Neuroscience Graduate \\ Program, University of California, San Francisco, California 94143
}

We characterized the dependence of motor learning in the monkey vestibulo-ocular reflex (VOR) on the duration, frequency, and relative timing of the visual and vestibular stimuli used to induce learning. The amplitude of the VOR was decreased or increased through training with paired head and visual stimulus motion in the same or opposite directions, respectively. For training stimuli that consisted of simultaneous pulses of head and target velocity $80-1000 \mathrm{msec}$ in duration, brief stimuli caused small changes in the amplitude of the VOR, whereas long stimuli caused larger changes in amplitude as well as changes in the dynamics of the reflex. When the relative timing of the visual and vestibular stimuli was varied, brief image motion paired with the beginning of a longer vestibular stimulus caused changes in the amplitude of the reflex alone, but the same image motion paired with a later time in the vestibular stimulus caused changes in the dynamics as well as the amplitude of the VOR. For training stimuli that consisted of sinusoidal head and visual stimulus motion, low-frequency training stimuli induced frequency-selective changes in the VOR, as reported previously, whereas high-frequency training stimuli induced changes in the amplitude of the VOR that were more similar across test frequency. The results suggest that there are at least two distinguishable components of motor learning in the VOR. One component is induced by shortduration or high-frequency stimuli and involves changes in only the amplitude of the reflex. A second component is induced by long-duration or low-frequency stimuli and involves changes in the amplitude and dynamics of the VOR.

Key words: motor learning; vestibulo-ocular reflex; dynamics; timing; eye movements; monkeys; oculomotor
The vestibulo-ocular reflex (VOR) stabilizes images on the retina during head turns by using vestibular signals to generate compensatory smooth eye movements in the opposite direction from head motion. Motor learning maintains the accuracy of the VOR by modifying the reflex whenever retinal image motion is associated persistently with head turns (Gonshor and Melvill Jones, 1973; Ito et al., 1974; Miles and Fuller, 1974; Gauthier and Robinson, 1975). Previous research has provided evidence for two sites of plasticity associated with motor learning in the VOR. One site is in the vestibular inputs to the cerebellar cortex of the floccular complex, and the other is in the vestibular inputs to neurons in the vestibular nucleus that receive direct monosynaptic inhibition from the floccular complex (Dufosse et al., 1978; Miles et al., 1980b; Watanabe, 1984; Lisberger and Pavelko, 1988; Lisberger, 1994; Lisberger et al., 1994b,c; Luebke and Robinson, 1994; Pastor et al., 1994; Partsalis et al., 1995).

With putative sites of plasticity established, a logical next step is to identify anatomical pathways, neural signals, and ultimately the cellular mechanisms that guide plasticity at these sites. The behavioral learning rule is well known. The conjunction of head turns and image motion causes motor learning in the VOR. If image motion is consistently in the same direction as head motion, then the amplitude of the VOR is too large, and learning causes

\footnotetext{
Received March 25, 1996; revised Sept. 16, 1996; accepted Sept. 19, 1996.

This work was supported by National Institutes of Health Grant EY10198 and a NASA Research Associate Fellowship to J.L.R. We thank M. Kahlon, V. Ferrera, G. Cohen, S. duLac, and M. Kvale for helpful comments on an earlier version of this manuscript.

Correspondence should be addressed to Jennifer L. Raymond, Department of Physiology, Box 0444, University of California San Francisco, San Francisco, CA 94143.

Copyright (C) 1996 Society for Neuroscience $0270-6474 / 96 / 167791-12 \$ 05.00 / 0$
}

it to decrease; if image motion is in the opposite direction from head motion, then the amplitude of the VOR is too small, and learning causes it to increase. Neural instantiation of the learning rule presumably involves the convergence of signals from vestibular and visual sensory pathways on sites of plasticity. Both putative sites of plasticity for the VOR receive the requisite convergence of visual and vestibular signals. The cerebellar cortex of the floccular complex receives vestibular inputs from second-order vestibular neurons in the vestibular nucleus and visual inputs over both the climbing fiber and mossy fiber pathways (Precht and Llinas, 1969; Simpson and Alley, 1974; Langer et al., 1985; Graf et al., 1988; Stone and Lisberger, 1990a,b). The vestibular nucleus receives inputs signaling head velocity from primary afferent and second-order vestibular neurons and highly processed or transformed vestibular inputs from the floccular complex and nucleus prepositus hypoglossi (Shimazu and Precht, 1966; Baker et al., 1972; Precht and Baker, 1972; Highstein, 1973; Baker and Berthoz, 1975; Ito et al., 1976, 1977; Lisberger and Pavelko, 1988; Lisberger et al., 1994a). It may receive visual inputs from the nucleus prepositus, the Purkinje cells, and the collaterals of climbing fibers that project to the floccular complex.

Three questions must be answered to advance our understanding of how visual and vestibular stimuli regulate cellular mechanisms of plasticity in the circuit for the VOR. First, which of the multiple visual and vestibular inputs to the sites of plasticity are involved in learning? Second, how are the sensory stimuli that drive learning represented in the firing patterns of those inputs? The neural signals that guide learning will not be exact replicas of the external visual and vestibular stimuli. For example, there is a $100 \mathrm{msec}$ latency difference for vestibular and visual signals to arrive at the sites of plasticity (Baker et al., 1969; Precht and 
Baker, 1972; Highstein, 1973; Miles et al., 1980a; Lisberger and Pavelko, 1988; Stone and Lisberger, 1990a,b; Lisberger et al., 1994b,c), and the signals also may be filtered or transformed in other ways. Third, how are the cellular mechanisms of plasticity regulated by electrical signals in their neural input pathways, and what transformations are performed in subcellular signaling pathways involved in plasticity?

In the present paper, we take a behavioral approach to these questions. We contend that we should be able to constrain the dynamic properties of the neural signals and cellular mechanisms that guide motor learning in the VOR through an examination of how learning is affected by systematic variation of the temporal properties of the sensory stimuli used to induce learning. Our analysis provides evidence for two components of learning in the VOR that are regulated by neural signals with different dynamics.

\section{MATERIALS AND METHODS}

Experiments were conducted on three male rhesus monkeys that had been trained to perform a visual fixation task to obtain liquid reinforcement, following the methods in Wurtz (1969). Using methods that have been described previously (Lisberger et al., 1994a), monkeys were anesthetized with isofluorane, and sterile procedure was used to implant bolts in the skull for restraining the head and to implant a coil of wire on one eye for measuring horizontal and vertical eye position. During experiments, each monkey sat in a specially designed primate chair to which his implanted head holder was secured. Vestibular stimuli were provided by a servo-controlled turntable (Contraves-Goertz, model 813) that rotated the monkey, the chair, and a set of 18 inch magnetic field coils together around a vertical axis.

VOR testing procedures and data analysis. We tested VOR performance by delivering passive head turns around a vertical axis in total darkness. At the beginning and end of each experiment, tests were run over a range of sinusoidal frequencies $(0.5,1,2,3,4,5,6,8$, and $10 \mathrm{~Hz})$, with amplitude $20^{\circ} / \mathrm{sec}$ peak-to-peak, and additional tests were run with pulse stimuli at a constant velocity of $15 \% \mathrm{sec}$ for $500-1000 \mathrm{msec}$. Each pulse stimulus consisted of a rapid acceleration from 0 to $15^{\circ} / \mathrm{sec}$, a period of constant velocity at $15 \% \mathrm{sec}$, and a deceleration back to $0 \% \mathrm{sec}$. The commanded acceleration was constant at $600^{\circ} / \mathrm{sec}^{2}$ for $25 \mathrm{msec}$, but the dynamics of the turntable resulted in accelerations that took $\sim 40 \mathrm{msec}$ to achieve peak velocity and had overshoots of $\sim 3^{\circ} / \mathrm{sec}$ (see Figs. 1, 2). In addition to the tests at the beginning and end of the experiment, the time course of learning was monitored at regular intervals during training by briefly placing the monkey in the dark and testing VOR performance using a small subset of the vestibular test stimuli. When the vestibular stimulus for testing the VOR was a pulse of head velocity, stimuli were delivered at an interval of $1.396 \mathrm{sec}$. During the intervals when the head was stationary between pulse stimuli, the monkey was rewarded for fixating a red light-emitting diode target projected $114 \mathrm{~cm}$ in front of him in the otherwise dark room. The target was extinguished $100 \mathrm{msec}$ before each vestibular pulse stimulus and was turned back on $100 \mathrm{msec}$ after each stimulus. When the vestibular stimulus for testing the VOR was a sine wave, the monkey was placed in darkness but was rewarded for keeping his eyes positioned within $\pm 10^{\circ}$ of straight-ahead gaze. Both of these procedures kept the eyes near straight-ahead gaze yet allowed measurement of the VOR in the dark. We did not record eye movements for attempted eccentric fixation with the head stationary, and therefore we cannot report whether our training conditions caused changes in the neural integrator, like those reported by Tiliket and colleagues (1994).

Voltages related to eye and head position were differentiated with an analog circuit to obtain signals related to eye and head velocity. Data were recorded on-line by a computer at $500 \mathrm{~Hz}$ per channel. The data were analyzed after the experiment by aligning the records and averaging eye and head velocity. Nearly all averages included 10 or more records, but in a few cases fewer than 10 records were averaged. For pulse stimuli, the records were aligned on the onset of head acceleration. Only saccadefree responses were included in the averages, and eye velocity records were edited before averaging to remove the rapid deflections caused by any saccades during the period of fixation before or after the vestibular stimulus (Lisberger et al., 1994a). The gain of the VOR was measured at various times during the pulse of head velocity as the averaged eye velocity divided by the imposed head velocity. For sinusoidal stimuli, the records were aligned on the zero crossings of head velocity. For frequen- cies $\leq 1 \mathrm{~Hz}$, eye velocity records were edited before averaging to remove the rapid deflections caused by saccades. For frequencies $>1 \mathrm{~Hz}$, analysis was limited to saccade-free cycles for which gaze position was within $10^{\circ}$ of straight-ahead gaze. Averaged eye and head velocity traces were subjected to Fourier analysis, and the gain of the VOR was estimated as the ratio of the fundamental components of eye and head velocity. The harmonic distortion of eye velocity was generally $<5 \%$.

Procedures for inducing learning. During a training period that lasted 3 $\mathrm{hr}$ (unless noted otherwise), learning was induced as the monkey viewed moving visual stimuli during passive whole-body rotation around a vertical axis. In some experiments the vestibular stimulus used to induce learning was a sine wave at a single frequency of $0.5,2,5,8$, or $10 \mathrm{~Hz}$ and with a peak-to-peak velocity of $20 \%$ s. In other experiments, the vestibular stimuli used to induce learning were pulses of head velocity. Each pulse consisted of a rapid acceleration from 0 to $15^{\circ} / \mathrm{sec}$, a period of constant velocity at $15^{\circ} / \mathrm{sec}$, and a deceleration back to $0^{\circ} / \mathrm{sec}$. The commanded acceleration was constant at $600 \% \mathrm{sec}^{2}$ for $25 \mathrm{msec}$, but in different experiments the duration of the constant velocity part of the pulse stimulus ranged from 30 to $950 \mathrm{msec}$, for total commanded pulse stimulus durations ranging from 80 to $1000 \mathrm{msec}$. Pulse stimuli were delivered at regular intervals of $1.096 \mathrm{sec}$, and the direction of the initial head acceleration was alternated so that one rightward and one leftward pulse of head velocity was delivered every $2.192 \mathrm{sec}$. This resulted in a trapezoidal head position profile (Fig. 1), with total excursions ranging from $0.8^{\circ}$ for pulses of head velocity with durations of $80 \mathrm{msec}$ to $14.6^{\circ}$ for pulses with durations of $1000 \mathrm{msec}$.

The visual motion stimulus for inducing learning in the VOR was provided by a high-contrast, black and white random-dot pattern that was reflected off a mirror galvanometer onto the back of a tangent screen 114 $\mathrm{cm}$ in front of the eyes. The visual stimulus subtended $16^{\circ}$ along the horizontal meridian and $11^{\circ}$ along the vertical meridian. Decreases in the amplitude of the VOR were produced by a combination of vestibular and visual stimuli called the "times zero" $(\times 0)$ stimulus condition. In the $\times 0$ condition, the visual stimulus moved in the same direction and at the same speed as the head, so that images would be stable on the retina if the eyes did not rotate within the head during vestibular stimuli. Thus, the ideal gain of the VOR (defined as the ratio of eye speed to head speed) would be zero. Increases in the gain of the VOR were produced with the "times two" $(\times 2)$ stimulus condition. In this case, the visual stimulus moved at the same speed but in the opposite direction from the head. Hence, the eye movements driven by the VOR needed to be twice the amplitude of the head movement to keep images stable on the retina, and the ideal gain of the VOR would be 2.0. Equivalently, for sinusoidal stimuli, the motion of the head and visual stimulus were in phase for the $\times 0$ stimulus condition and $180^{\circ}$ out of phase for the $\times 2$ stimulus condition. For $500 \mathrm{msec}$ and $1000 \mathrm{msec}$ pulse stimuli, the visual stimulus began at an eccentric position $\left(3^{\circ}\right.$ or $7^{\circ}$, respectively), so that its movement would not take it beyond the edge of the tangent screen. To maintain a constant level of alertness and to keep the stimulus roughly centered in the visual field during vestibular stimulation, monkeys were rewarded at intervals of $1.5-4.0 \mathrm{sec}$ for keeping their gaze within $\pm 10^{\circ}$ of the center of the visual stimulus. In some experiments, stroboscopic illumination (50 $\mathrm{Hz}$ ) was used to project the visual stimulus onto the mirror galvanometer. This allowed for rapid transition from visual stimulation to total darkness.

Experiments with $\times 0$ and $\times 2$ training stimuli were alternated, and experiments were separated by at least $48 \mathrm{hr}$ to allow the gain to readapt to its normal value before each experiment. To test for residual learning from previous training sessions, we performed statistical analysis of the pretraining gain values of monkeys $\mathrm{A}$ and $\mathrm{D}$, for which there were complete data sets. For the data of Figure 3, a three-factor ANOVA of the pretraining gain of the VOR measured at two time points (40-50 and 425-475 msec after onset of head motion) revealed no consistent variation among experiments with different training stimulus durations $\left(F_{(4,20)}=1.50 ; p<0.24\right)$ or between experiments with $\times 0$ versus $\times 2$ visual conditions $\left(F_{(1,20)}=0.31 ; p<0.58\right)$. Furthermore, there was no significant difference in the prelearning gain at the early and late time points $\left(F_{(1,20)}=0.13 ; p<0.72\right)$, and there were no significant interaction effects among any of the factors. Similarly, for the data of Figure 6, a three-factor ANOVA revealed no consistent variation in the pretraining gain of the VOR among experiments with differently timed visual stimuli $\left(F_{(2,12)}=2.84 ; p<0.10\right)$, between experiments with $\times 0$ versus $\times 2$ visual conditions $\left(F_{(1,12)}=0.23 ; p<0.64\right)$, or between measurements taken $40-50$ versus $450-575 \mathrm{msec}$ after the onset of head motion $\left(F_{(1,12)}=0.66\right.$; $p<0.43$ ), and there were no significant interaction effects. For the data of Figure 7, a three-factor ANOVA revealed no consistent variation in 

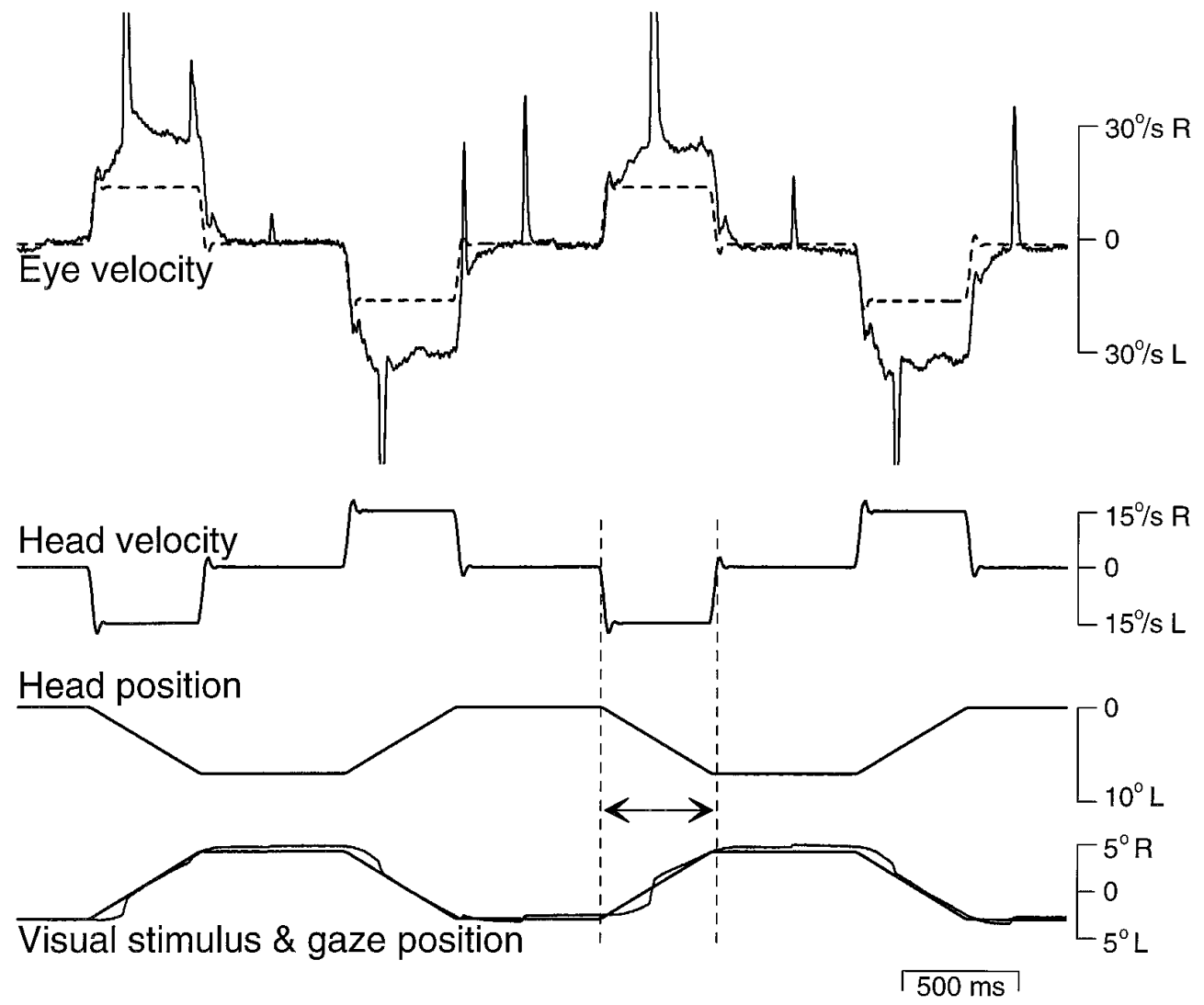

Figure 1. Example of a $\times 2$ training stimulus used to induce motor learning in the VOR. Eye velocity is plotted relative to the head. Head velocity, head position, visual stimulus position, and gaze position are plotted relative to the stationary world. Gaze position was computed as the sum of head position in the world plus eye position in the head. In all traces, up represents rightward position or motion $(R)$; down represents leftward position or motion $(L)$. The amplitude of saccadic eye velocities has been cropped. Arrows indicate the duration of the pulses of head and visual stimulus movement, which varied in different experiments.

the pretraining gain of the VOR among experiments with different training frequencies $\left(F_{(4,90)}=0.37 ; p<0.83\right)$ or between experiments with $\times 0$ versus $\times 2$ visual conditions $\left(F_{(1,90)}=0.93 ; p<0.34\right)$. There was a significant difference in the prelearning gain measured with different test frequencies $\left(F_{(8,90)}=2.77 ; p<0.01\right)$, as expected from previous reports (Keller, 1978). There were no significant interaction effects.

\section{RESULTS}

\section{The duration of individual training stimuli affects learning}

In the first set of experiments, we used pulses of head and visual stimulus velocity as the training stimulus and examined the effect of varying the duration of the pulse on learned changes in the VOR. Pulse durations ranged from 80 to $1000 \mathrm{msec}$, and learning was induced by either $\times 0$ or $\times 2$ stimulus conditions. Figure 1 illustrates training with a $500 \mathrm{msec}, \times 2$ stimulus. The head and visual stimulus were initially stationary and then simultaneously began moving at the same speed but in opposite directions (head left, visual stimulus right). After they moved for $500 \mathrm{msec}$ at a speed of $15 \%$ sec, both the head and visual stimulus stopped. Then, $1.096 \mathrm{sec}$ after the onset of the previous stimulus, the head and visual stimulus moved with the same pulse trajectory in directions opposite from the previous movement (head right, visual stimulus left). The eye velocity during training resulted from a combination of eye movements driven by the VOR, predicted by the dashed trace, and visually guided tracking eye movements that attempted to match gaze position and velocity to target position and velocity.

Figure $2 A$ illustrates the VOR response elicited by a $500 \mathrm{msec}$ pulse of head velocity in darkness and its modification by $3 \mathrm{hr}$ of training with $150 \mathrm{msec}$ pulses of head and visual stimulus velocity in the $\times 2$ training condition. Before training (dashed trace), the VOR response consisted of a $500 \mathrm{msec}$ pulse of eye velocity that was opposite in direction from and nearly equal in amplitude to the vestibular stimulus. During the $450 \mathrm{msec}$ period of constant head velocity, the evoked eye velocity remained nearly constant. After training (heavy solid trace), the eye velocity response was larger than control throughout the $450 \mathrm{msec}$ interval of constant head velocity. Thus, when tested with long vestibular stimuli, the learned changes in the VOR induced by training with brief pulses of visual-vestibular stimulation were expressed at times in the test stimulus that were well beyond the duration of the training stimulus. Furthermore, the changes were approximately equal in amplitude at all times during the vestibular stimulus.

Figure $2 B$ illustrates the learned changes in the VOR induced by $1000 \mathrm{msec}$ pulses of head and visual stimulus velocity in the $\times 2$ training condition. During the $3 \mathrm{hr}$ training period, the same number of visual-vestibular stimuli were delivered as in the experiment of Figure $2 A$. Again, the VOR was tested with $500 \mathrm{msec}$ pulses of head velocity in the dark, so the responses in Figure 2, $A$ and $B$, are directly comparable. Comparison of the VOR evoked after training (heavy solid trace) with the control response (dashed trace) reveals that the $1000 \mathrm{msec}$ training stimulus induced a large change in the amplitude of the VOR. Furthermore, the evoked eye velocity increased throughout the test stimulus, in contrast to the eye movements elicited before training or after training with the $150 \mathrm{msec}$ stimulus.

A comparison of the learned components of the VOR (Fig. 2, bottom traces) highlights the different effects of training with brief and long stimuli. These traces were obtained by subtracting the eye velocity evoked by the vestibular test stimulus before training from that evoked after training. When the training stimulus had a duration of $150 \mathrm{msec}$, the learned component of the VOR had a roughly constant amplitude throughout the test stimulus. When the training stimulus had a duration of $1000 \mathrm{msec}$, the learned 
A

Figure 2. Examples of learned changes in the VOR induced by training with pulses of head and visual stimulus velocity that were $150 \mathrm{msec}(A)$ or $1000 \mathrm{msec}$ $(B)$ in duration. Dashed eye velocity trace: VOR before learning; heavy solid trace: VOR after learning. Each trace is the average of at least ten responses, and fine traces indicate the standard deviation of the averaged eye velocity after learning. The learned component of the VOR was computed by subtracting the eye movement response elicited before learning from the eye movement response elicited after learning.
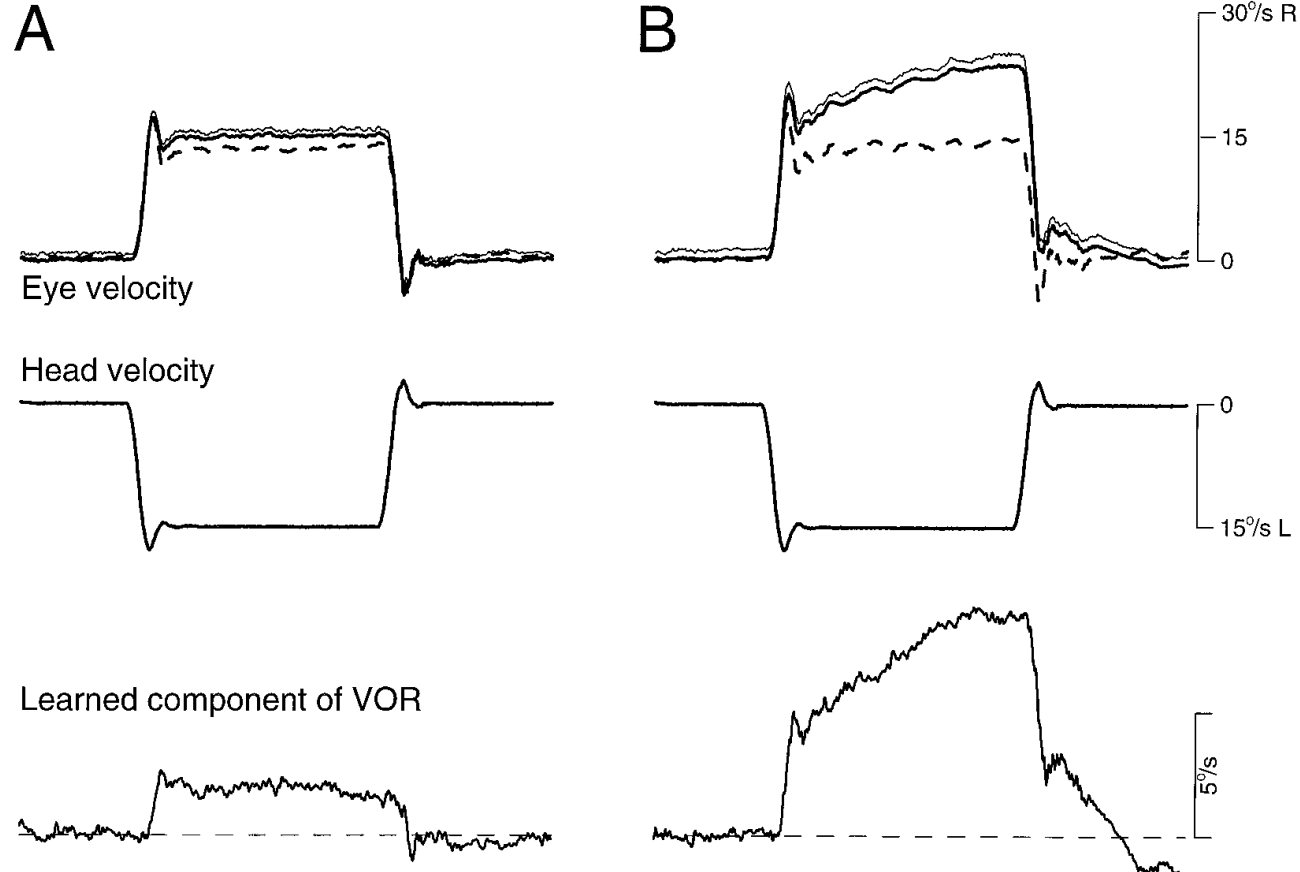

component increased in amplitude throughout the test stimulus, reflecting changes in both the amplitude and time course or dynamics of the eye movement evoked by the test stimulus.

We performed a quantitative analysis of the changes in the gain of the VOR two times during the test stimulus: (1) during the interval from 40 to $50 \mathrm{msec}$ after the onset of the vestibular stimulus and (2) during the interval from 425 to $475 \mathrm{msec}$ after the onset of the vestibular stimulus. The pretraining values of the gain of the VOR were $1.01 \pm 0.02$ and $0.98 \pm 0.01$ (mean \pm SEM) for the early and late intervals, respectively. Learned changes in the amplitude of the VOR are expressed as the ratio of the gain of the VOR after training divided by the gain of the VOR before training. Values $<1.0$ represent a learned decrease in the gain of the VOR, and values $>1.0$ represent a learned increase in the gain.

Each graph in Figure 3 plots learned changes in the gain of the VOR as a function of the duration of the training stimulus for both $\times 0$ (open symbols) and $\times 2$ (closed symbols) training conditions. Each point represents the results from $3 \mathrm{hr}$ of exposure to the training stimulus during one experimental session, and different symbols (circles, squares, triangles) represent the results from different monkeys. In each case, the test stimulus used to measure the VOR was a $500 \mathrm{msec}$ pulse of head velocity at $15^{\circ} / \mathrm{sec}$. In Figure $3 A$, the gain of the VOR was estimated by averaging eye and head velocity over the interval from 40 to $50 \mathrm{msec}$ after the onset of the vestibular test stimulus. For all durations of training stimulus tested, changes in the gain of the VOR in this early interval were adaptive: training with $\times 0$ stimuli produced a decrease in the gain of the VOR, and training with $\times 2$ stimuli produced an increase in the gain. The changes were small, however, and increased only slightly as a function of the duration of the training stimulus. In the interval from 425 to $475 \mathrm{msec}$ after the onset of head motion (Fig. $3 B$ ), the learned changes in the gain of the VOR were larger and showed a strong relationship to the duration of the training stimulus. Furthermore, comparison of Figure 3, $A$ and $B$, reveals that training with a long-duration training stim-
Figure 3. Quantitative analysis of learned changes in the gain of the VOR induced by training stimuli of different durations. Changes in gain are plotted as the ratio of the gain after learning to the gain before learning. $A$, Early VOR: the gain ratio was measured $40-50 \mathrm{msec}$ after the onset of head movement. $B$, Late VOR: the gain ratio was measured 425-475 msec after the onset of head movement. Each data point represents the results from one training session. Open symbols, $\times 0$ stimulus conditions; filled symbols, $\times 2$ stimulus conditions. Circles, Monkey A; squares, monkey D; triangles, monkey E.
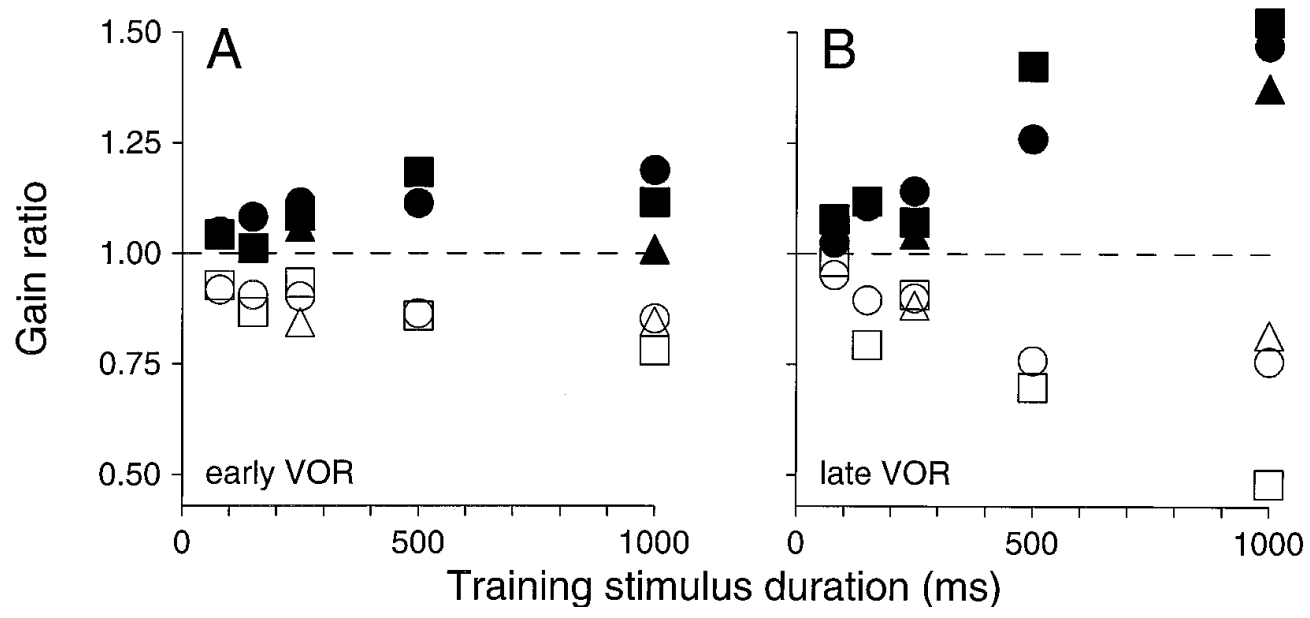


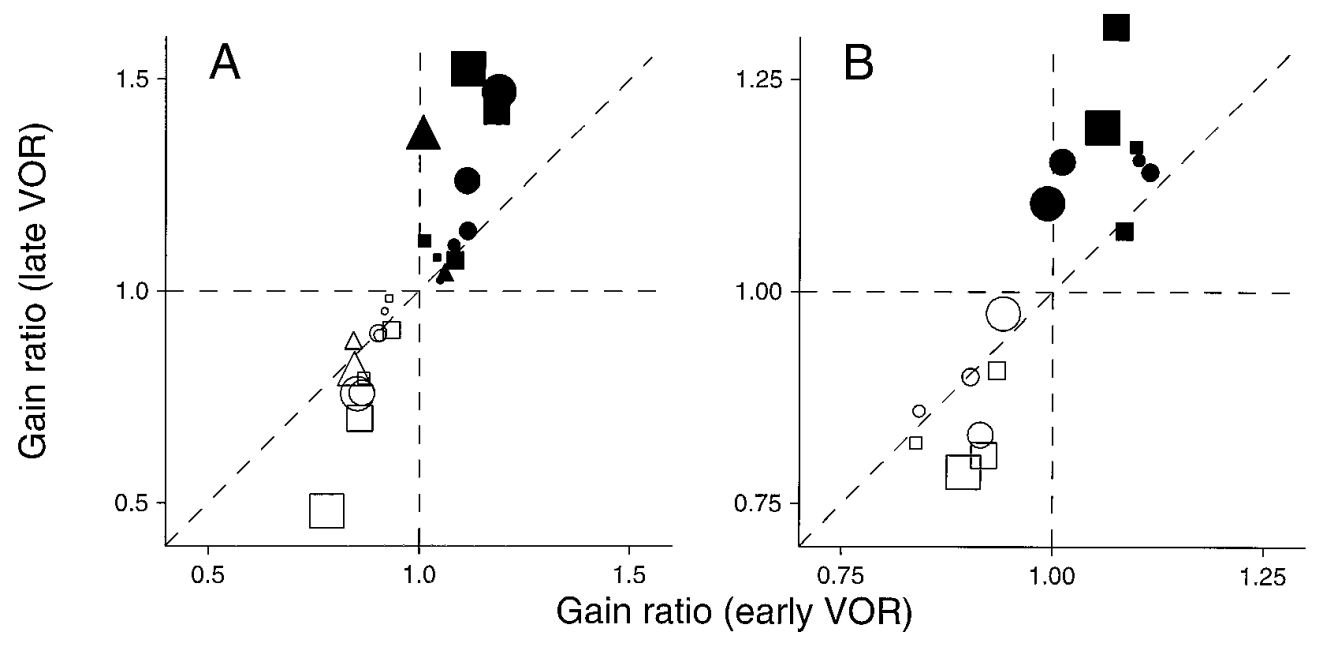

Figure 4. Comparison of changes in the dynamics of the VOR induced by training stimuli of different durations. The change in gain of the late VOR (gain ratio measured $425-475 \mathrm{msec}$ after the onset of head motion) is plotted relative to the change in gain of the early VOR (gain ratio measured $40-50 \mathrm{msec}$ after the onset of head motion). The size of the symbol indicates the duration of the training stimulus. Smallest symbols represent the results of training with $80 \mathrm{msec}$ stimuli. Progressively larger symbols represent the results of training with $150,250,500$, and $1000 \mathrm{msec}$ stimuli. $A$, The training period for each experiment was $3 \mathrm{hr}$, so that the total duration of visual-vestibular stimulation during training varied from $\sim 800$ sec for $80 \mathrm{msec}$ stimuli to $10,000 \mathrm{sec}$ for $1000 \mathrm{msec}$ stimuli. $B$, The duration of the training period was adjusted to compensate for the durations of the individual stimuli, so that total duration of visualvestibular stimulation was $2500 \mathrm{sec}$. Note the difference in scale in $A$ and $B$. Open symbols, $\times 0$ stimulus conditions; filled symbols, $\times 2$ stimulus conditions. Circles, Monkey A; squares, monkey D; triangles, monkey E. ulus (e.g., 500 or $1000 \mathrm{msec}$ ) induced bigger changes late rather than early in the VOR.

In Figure $4 A$, we have replotted the data from Figure 3 in a way that directly compares the ratio of the post- and pretraining gains of the VOR in the $425-475 \mathrm{msec}$ interval with that in the early (40-50 msec) interval. Each symbol represents the results of a single $3 \mathrm{hr}$ training session, and the size of the symbol indicates the duration of the training stimulus. The smallest symbols represent the changes produced by $80 \mathrm{msec}$ training stimuli, and progressively larger symbols represent the results of training with 150, 250, 500, and $1000 \mathrm{msec}$ stimuli. The smaller symbols are clustered near the dashed diagonal line, indicating equal changes in the gain of the early and late components of the VOR. In contrast, the larger symbols tend to plot closer to the dashed vertical line, indicating larger changes in the late VOR rather than the early VOR. These differential changes in the gain of the VOR measured early and late during the $500 \mathrm{msec}$ test stimulus represent a change in the dynamics of the VOR after training with long-duration stimuli.

The results in Figures 3 and $4 A$ suggest that there is a relationship between the induction of learned changes in the dynamics of the VOR and the temporal properties of the individual stimuli used to induce learning. Because the same number of stimuli were delivered in each experiment, however, the total duration of visual-vestibular stimulation varied with the duration of the individual stimuli. In a $3 \mathrm{hr}$ training session, the total duration of stimulation was $\sim 800 \mathrm{sec}$ with $80 \mathrm{msec}$ stimuli, $1500 \mathrm{sec}$ with $150 \mathrm{msec}$ stimuli, $2500 \mathrm{sec}$ with $250 \mathrm{msec}$ stimuli, $5000 \mathrm{sec}$ with $500 \mathrm{msec}$ stimuli, and 10,000 sec with $1000 \mathrm{msec}$ stimuli. Hence, we needed to address the possibility that the apparent effects of the duration of the individual training stimuli were related more directly to the total duration of visual-vestibular stimulation during training. We therefore conducted a series of experiments that induced learning with training stimulus pulses of different durations, as in Figures 3 and $4 A$, but with the duration of each training session adjusted so that all experiments provided a total of $\sim 2500 \mathrm{sec}$ of visual-vestibular stimulation. Figure $4 B$ uses the same graphical form as Figure $4 A$ to compare the changes in the early and late VOR produced by 45 min of training with stimuli that were $1000 \mathrm{msec}$ in duration ( $\sim 2500$ training stimuli), $90 \mathrm{~min}$ of training with $500 \mathrm{msec}$ stimuli ( $\sim 5000$ stimuli), $180 \mathrm{~min}$ of training with $250 \mathrm{msec}$ stimuli $(\sim 10,000$ stimuli), and $300 \mathrm{~min}$ of training with $150 \mathrm{msec}$ stimuli ( 16,500 stimuli). Pulse durations of $80 \mathrm{msec}$ were excluded from this control experiment, because they would have required almost $10 \mathrm{hr}$ of training.

In general, even with the total duration of visual-vestibular stimulation during training held constant, 150 and $250 \mathrm{msec}$ stimuli (Fig. 4B, small symbols) produced changes in the late and early components of the VOR that were similar in amplitude and therefore plotted near the dashed diagonal line of slope 1. In contrast, 500 and $1000 \mathrm{msec}$ stimuli (Fig. 4B, large symbols) produced bigger changes in the late than in the early VOR and therefore plotted closer to the vertical rather than the horizontal dashed line. A single exception to this general finding was the experiment in which monkey A received 45 min of training with a $1000 \mathrm{msec} \times 0$ stimulus (large open circle), and the change in the late VOR was smaller than the change in the early VOR. In this case, however, changes in both the late and early VOR were quite small, indicating little overall learning. This one data point indicates that learned changes in the gain and dynamics of the VOR can be small or fail to occur if the total number of stimuli is low or the total length of the training session is very short. Indeed, the smaller size of the learned changes made it practical to use different scales in Figure 4, $A$ and $B$. Nevertheless, to the extent that learning occurs, the duration of the individual stimuli used in training seems to be a key factor in determining the extent to which the dynamics of the VOR are modified by learning. Neither the length of the training session and number of training stimuli (held constant in Fig. $4 A$ ) nor the total duration of visual-vestibular stimulation (held constant in Fig. $4 B$ ) can account for the induction of changes in the dynamics of the VOR. 

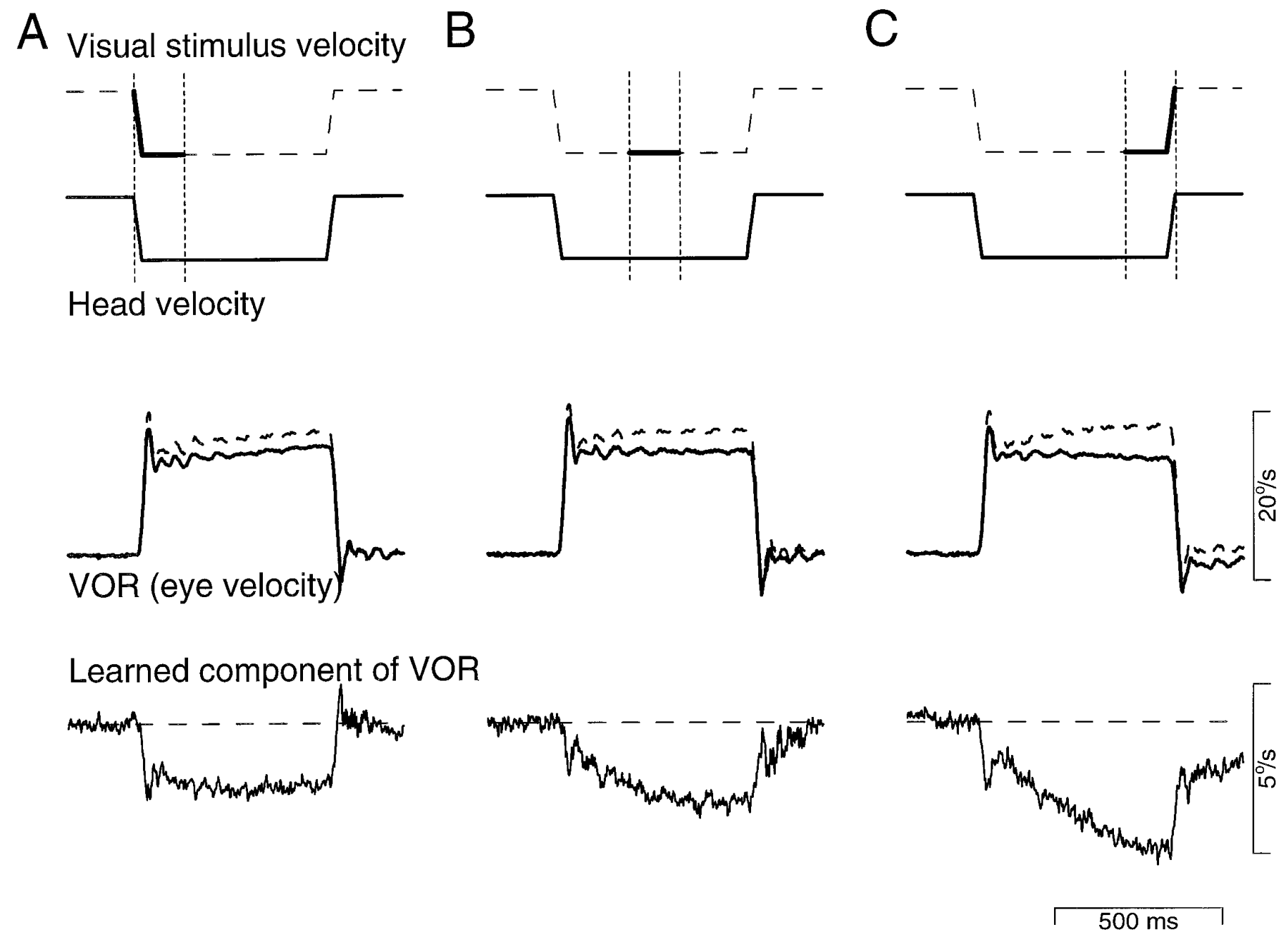

Figure 5. Examples of changes in the VOR induced by a $150 \mathrm{msec}$ period of $\times 0$ visual stimulus motion that was paired with the beginning $(A)$, middle $(B)$, or end $(C)$ of a $600 \mathrm{msec}$ pulse of head motion. Top traces indicate schematically the training stimuli used to induce learning. Dashed portions of the visual stimulus velocity traces indicate periods of darkness; solid portions indicate periods during which the visual stimulus was illuminated with stroboscopic light. Middle traces show the VOR responses to a $600 \mathrm{msec}$ pulse of head velocity before (dashed traces) and after (solid traces) 3 hr of exposure to the training stimulus. Bottom traces show the learned component of the VOR, obtained by subtracting the eye velocity elicited by the vestibular test stimulus before learning from the eye velocity elicited after learning.

\section{The timing of image motion during head turns affects learning}

Because training stimuli of different durations induce different learned changes in the VOR, the neural representations of the training stimuli at the sites of plasticity must have dynamics that can affect the plasticity mechanism. Because the duration of the visual and vestibular stimuli were varied together in the above experiments, the dynamics of the signals important for learning could have resulted from the dynamics of signals related to either of these components of the training stimulus. To distinguish between these possibilities, we conducted an additional experiment that varied the relative timing of the two stimuli during training. The logic behind the experiment was that if the neural representation of the vestibular stimulus at the site of plasticity changes with time, then different changes might be induced in the VOR depending on when the visual stimulus was presented relative to the vestibular stimulus.

The training stimuli used in this experiment are represented schematically in the top traces of Figure 5. The vestibular stimulus used during training was always a $600 \mathrm{msec}$ pulse of head velocity $\left(15^{\circ} / \mathrm{sec}\right)$. Learning was induced by a short period of $\times 0$ (as shown in Fig. 5) or $\times 2$ visual stimulation that was provided only during the first, middle, or last $150 \mathrm{msec}$ of the vestibular stimulus. This provided visual stimulation during intervals that were $0-150$, $225-375$, or $450-600 \mathrm{msec}$ after the onset of the vestibular stimulus. We accomplished a rapid switch between visual stimulation in one part of the vestibular stimulus and total darkness during the rest of the vestibular stimulus by using $50 \mathrm{~Hz}$ stroboscopic illumination to project the visual stimulus. In several control experiments, $50 \mathrm{~Hz}$ stroboscopic illumination produced changes in the VOR similar to those induced by continuous illumination when otherwise identical stimulus paradigms were used to induce learning (data not shown).

The eye velocity traces in Figure 5 illustrate the changes in the VOR that occurred when $\times 0$ visual stimuli were presented at different times during the vestibular stimulus used for training. When learning was induced by pairing the visual stimulus with the beginning of the vestibular stimulus (Fig. $5 A$ ), there was a decrease in the gain of the VOR that was similar in amplitude in the early and late phases of the VOR response. When learning was 


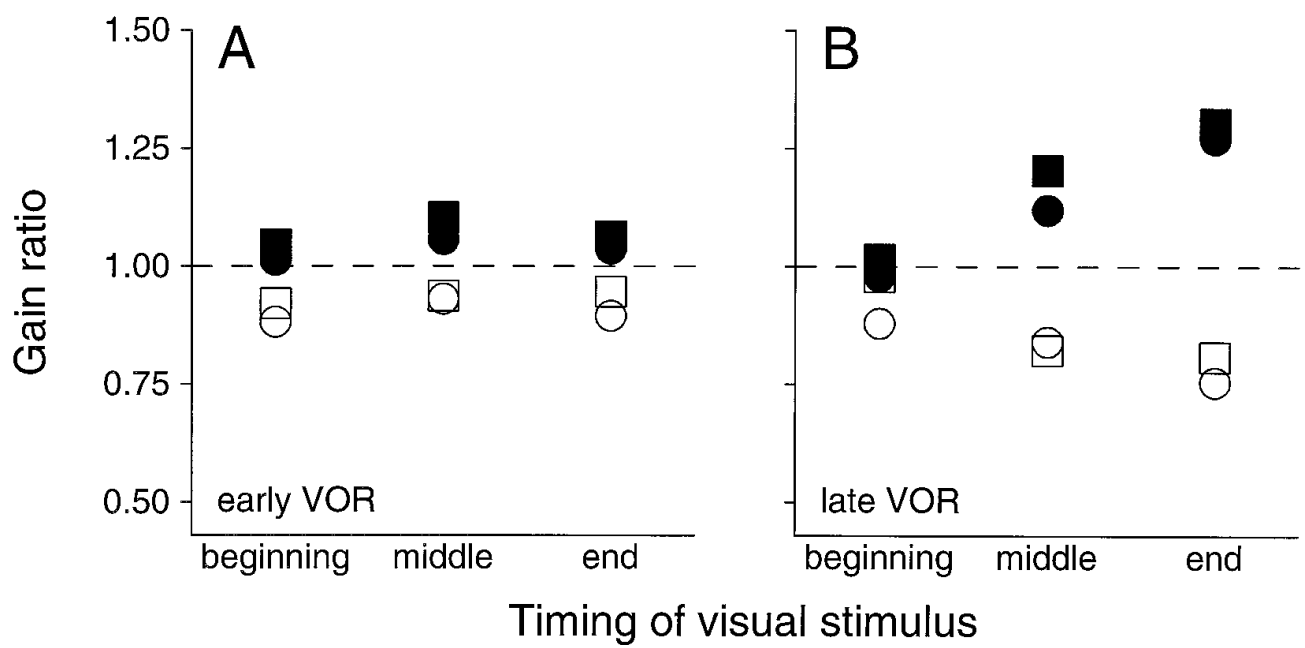

Figure 6. Summary of learned changes in the VOR induced by $150 \mathrm{msec}$ of visual stimulus motion paired with the beginning, middle, or end of a $600 \mathrm{msec}$ vestibular stimulus. Changes in the gain of the VOR are plotted as the ratio of the gain after learning to the gain before learning. $A$, Early VOR, measured $40-50$ msec after the onset of head motion. $B$, Late VOR, measured 450-575 msec after the onset of head motion. Open symbols, $\times 0$ stimulus conditions; filled symbols, $\times 2$ stimulus conditions. Circles, Monkey A; squares, monkey D. induced by pairing the visual stimulus with the middle (Fig. $5 B$ ) or last (Fig. 5C) $150 \mathrm{msec}$ of the vestibular stimulus, there were changes in the dynamics as well as the gain of the VOR. The dependence of the learned changes in the dynamics of the VOR on the timing of the visual stimulus relative to the vestibular stimulus is most apparent in a comparison of the learned components of the VOR for the three different training stimuli (Fig. 5, bottom traces).

On average, the pretraining values of the gain of the VOR were $0.98 \pm 0.01$ and $0.99 \pm 0.01$ (mean \pm SEM) for early (40-50 $\mathrm{msec})$ and late $(425-575 \mathrm{msec})$ measurement intervals, respectively; however, we observed some monkey-to-monkey and dayto-day variability in the prelearning dynamics of the VOR as tested with $500 \mathrm{msec}$ pulses of head velocity. For example, the prelearning eye velocity traces in Figure 5, $A$ and $C$, show increasing eye velocity during the constant velocity vestibular test stimuli. In other training sessions, the prelearning VOR response was constant in velocity or exhibited a slight deceleration during the constant velocity test stimulus. As detailed in Materials and Methods, statistical analysis revealed no consistent variation in the prelearning VOR dynamics that could account for the changes in dynamics induced by different training conditions. Furthermore, different training stimuli could induce different changes in dynamics even when the prelearning VOR responses were similar. For example, the prelearning VOR responses in Figure $5 A, C$ both increase in velocity during the test stimulus, but exposure to one training stimulus resulted in a postlearning response that decreased in velocity during the stimulus (Fig. $5 C$ ), whereas exposure to the other training stimulus resulted in a postlearning response that increased in velocity like the prelearning response (Fig. 5A).

Figure 6 summarizes the results from two monkeys (circles and squares) for both $\times 0$ (open symbols) and $\times 2$ (closed symbols) stimulus conditions. Results are shown for training with the visual stimulus present during the beginning $(0-150 \mathrm{msec})$, middle ( $225-$ $375 \mathrm{msec})$, or end $(450-600 \mathrm{msec})$ of the vestibular stimulus. Changes in gain are plotted as the ratio of the gain of the VOR after training to that before training

Learned changes in the early part of the VOR, measured 40-50 msec after the onset of head motion in the dark (Fig. 6A) were generally small and did not depend strongly on when the visual stimulus was delivered relative to the vestibular stimulus during training. In contrast, learned changes in the later phase of the VOR, measured $450-575 \mathrm{msec}$ after the onset of head motion in the dark (Fig. 6B), were biggest if learning was induced by presenting the visual stimulus at the end of the $600 \mathrm{msec}$ vestibular stimulus. This pattern of changes in the early and late VOR reflects changes in dynamics when the visual stimulus was paired with the end of the vestibular stimulus, smaller changes in dynamics when the visual stimulus was paired with the middle of the vestibular stimulus, and changes in gain alone when the visual stimulus was paired with the beginning of the vestibular stimulus.

Additional experiments were performed on one monkey to control for the possibility that the effect of changing the timing of the visual stimulus during training was related to the presence of an acceleration or deceleration during the period of visual stimulation for some of the training stimuli in Figures 5 and 6. In these control experiments, the visual stimulus was presented during different times in the constant velocity part of the vestibular stimulus, either from 50 to $200 \mathrm{msec}$ or from 400 to $550 \mathrm{msec}$ after the onset of the vestibular stimulus. Visual stimuli presented at 50-200 msec produced relatively similar changes in the early and late VOR; gain ratios for the early versus late VOR were 0.85 versus 0.79 for $\times 0$ training, and were 1.08 versus 1.10 for $\times 2$ training. In contrast, visual stimuli presented at $400-550 \mathrm{msec}$ produced a bigger change in the late than in the early VOR; gain ratios for the early versus late VOR were 0.94 versus 0.81 for $\times 0$ training and 1.03 versus 1.36 for $\times 2$ training. Thus, visual image motion that occurred during later times in the constant velocity part of the vestibular stimulus induced changes in the dynamics as well as the gain of the VOR, whereas image motion that occurred during early times in the constant velocity part of the vestibular stimulus induced primarily changes in gain.

\section{The frequency of sinusoidal training stimuli affects learning}

In a companion series of experiments, learning was induced in the VOR with continuous, sinusoidal head and visual stimulus motion. For each experiment, the training stimulus consisted of sinusoidal oscillation at a single frequency, and head and visual stimulus motion were either in phase $(\times 0)$ or out of phase $(\times 2)$. Before and after training, the VOR was tested with continuous sinusoidal head rotations in darkness over a range of frequencies from 0.5 to $10 \mathrm{~Hz}$ and with peak-to-peak amplitude of $20^{\circ} / \mathrm{sec}$. The effects of training were assessed by computing the ratio of the gain of the VOR after training to that before training and plotting this gain ratio as a function of the frequency of the test stimulus.

Each panel in Figure 7 plots the results for training at a single 
Figure 7. Learned changes in the gain of the VOR induced by sinusoidal training stimuli. Each plot shows the results for a single training frequency $(0.5,2,5$, 8 , or $10 \mathrm{~Hz}$, indicated at the top of the plot). Changes in gain (post-training/pretraining gain ratio) are plotted as a function of the frequency of the sinusoidal vestibular test stimuli used to measure the VOR. Open symbols, $\times 0$ stimulus conditions; filled symbols, $\times 2$ stimulus conditions. Circles, Monkey A; squares, monkey $\mathrm{D}$; triangles, monkey $\mathrm{E}$.

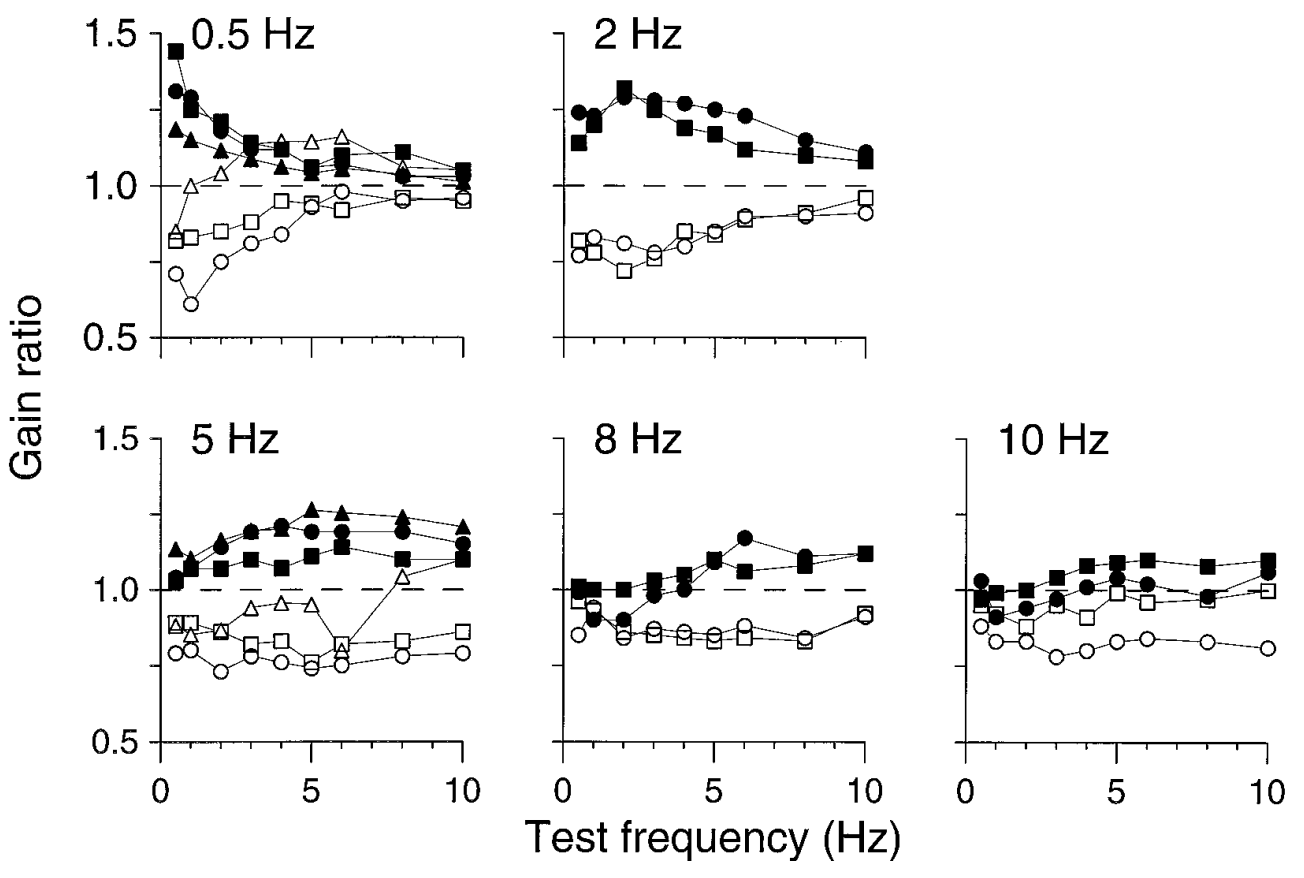

frequency, indicated at the top of each plot. After training with $\times 0$ or $\times 2$ stimuli at $0.5 \mathrm{~Hz}$, learned changes in the gain of the VOR were frequency-selective. The changes were biggest at test frequencies near $0.5 \mathrm{~Hz}$ and were progressively smaller when tested at higher frequencies, with little change at test frequencies of 8 and $10 \mathrm{~Hz}$. Similarly, training with $2 \mathrm{~Hz}$ stimuli produced changes in the gain of the VOR that were biggest at frequencies close to $2 \mathrm{~Hz}$. These results for training with sinusoidal stimuli at low frequencies confirmed previous findings of frequency-selective changes in the gain of the VOR (Collewijn and Grootendorst, 1979; Godaux et al., 1983; Lisberger et al., 1983; Powell et al., 1991); however, the frequency-selectivity of learned changes in the VOR was less evident at higher training frequencies. Training at $5 \mathrm{~Hz}$ still induced adaptive changes in the gain of the VOR, but the changes were similar across test frequency. Training with the highest-frequency stimuli $(8$ and $10 \mathrm{~Hz})$ produced smaller and less consistent changes in the gain of the VOR, although the changes generally were in the adaptive direction.

Figure 8 plots the effect of $\times 0$ and $\times 2$ training on the phase of the VOR for the experiments in Figure 7. Change in phase is plotted as the phase of eye velocity relative to head velocity after learning minus the phase of eye velocity relative to head velocity before learning. Small changes in phase were observed after training, and these changes were consistent across monkeys, although they never exceeded $10^{\circ}$. After training with $\times 2$ stimuli, there was an increase in phase lead at the lower test frequencies and an increase in phase lag at the higher test frequencies. After training with $\times 0$ stimuli, there was a increase in phase lag at the lower test frequencies and an increase in phase lead at the higher test frequencies. For $0.5,2$, and $5 \mathrm{~Hz}$ training stimuli, the cross-
Figure 8. Changes in the phase of the VOR induced by sinusoidal training stimuli. Changes in the phase of eye velocity relative to head velocity are plotted as a function of the frequency of the sinusoidal vestibular test stimuli used to measure the VOR. Open symbols, $\times 0$ stimulus conditions; filled symbols, $\times 2$ stimulus conditions. Circles, Monkey A; squares, monkey $\mathrm{D}$; triangles, monkey E.
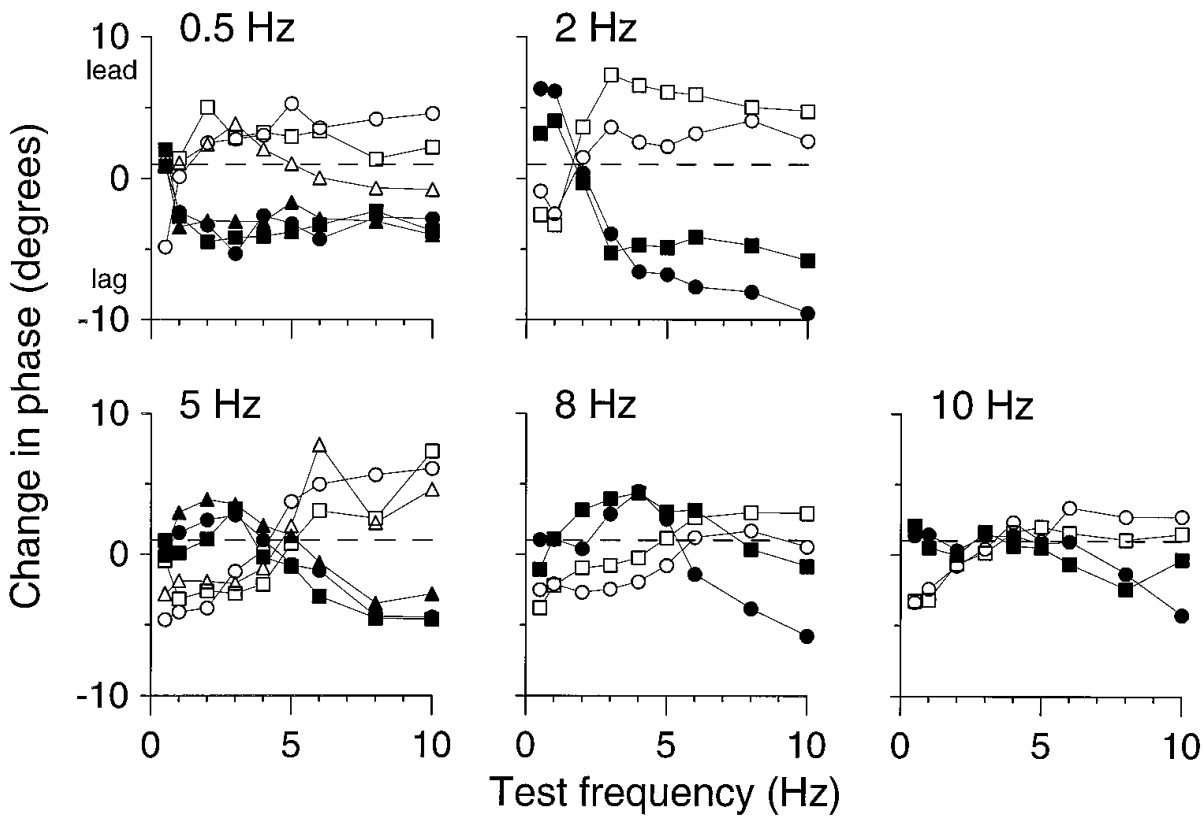
over from increased phase lead to increased phase lag occurred close to the training frequency. This is consistent with previous reports for training frequencies $\leq 2 \mathrm{~Hz}$ (Godaux et al., 1983; Lisberger et al., 1983; Powell et al., 1991). For 8 and $10 \mathrm{~Hz}$ training stimuli, however, the phase crossover occurred at a test frequency below the training frequency.

The differential effects on the gain and dynamics of the VOR induced by training with sinusoidal visual-vestibular stimuli of different frequencies paralleled the effects induced by stimuli of different durations. Like the long-duration visual-vestibular stimulus pulses, low-frequency sinusoidal stimuli induced a change in the dynamics as well as the amplitude of the VOR, as evidenced by the differential changes in gain across test frequency. Like the brief visual-vestibular stimulus pulses, high-frequency sinusoidal stimuli induced a change in the amplitude of the VOR with little differential effect across frequency. Converted to the time domain, the absence of a differential effect across frequency corresponded to little effect on the dynamics of the reflex.

For a more direct comparison of the effects observed in the time and frequency domains, we tested the VOR with sinusoidal vestibular stimuli before and after training with short- and longduration pulses of visual-vestibular stimulation. After training with 1000 msec stimuli (Fig. 9A), changes in the gain of the VOR were biggest when tested at low frequencies, but after training with 250 msec stimuli (Fig. 9B), changes were similar across test frequency. The estimated power spectra of the short and long vestibular pulse stimuli are shown in Figure 9, $A 2$ and B2. Power is normalized to the power contained in a single sinusoidal stimulus with an amplitude of $20 \%$ sec peak-to-peak, i.e., power is normalized to the power contained in each of the sinusoidal training stimuli used to induce learning in the experiments of Figures 7 and 8 .

The spectral analysis revealed that for the $1000 \mathrm{msec}$ stimuli, most of the power was at $0.5 \mathrm{~Hz}$ and the power at $0.5 \mathrm{~Hz}$ was more than twice that contained in the $0.5 \mathrm{~Hz}$ sinusoidal training stimuli of Figures 7 and 8. This is consistent with the $1000 \mathrm{msec}$ training stimuli having effects on the VOR measured with sinusoidal vestibular stimuli that are similar to those of the $0.5 \mathrm{~Hz}$ training stimuli. The $250 \mathrm{msec}$ stimuli contained less power overall than the $1000 \mathrm{msec}$ stimuli (compare scale in Fig. 9, $A_{2}$ and $B_{2}$ ), because the $250 \mathrm{msec}$ stimuli were shorter in duration than the $1000 \mathrm{msec}$ stimuli. The largest peak was near $0.5 \mathrm{~Hz}$, because the interval for repeat of the stimulus was $2.192 \mathrm{sec}$; however, the 250 msec stimuli contained considerably less power at $0.5 \mathrm{~Hz}$ than the $1000 \mathrm{msec}$ stimuli or the $0.5 \mathrm{~Hz}$ sinusoidal training stimuli. Furthermore, the $250 \mathrm{msec}$ stimuli contained proportionately more power at higher frequencies than the $1000 \mathrm{msec}$ stimuli. Despite the power they contained at $0.5 \mathrm{~Hz}$, the effects of the $250 \mathrm{msec}$ training stimuli on the VOR measured with sinusoidal vestibular stimuli were similar to the effects of high-frequency sinusoidal training stimuli. This may have been because the power at $0.5 \mathrm{~Hz}$ was below the threshold for induction of the low-frequency component of learning, or it may have been attributable to some complex interaction of the low and higher frequencies contained in the $250 \mathrm{msec}$ training stimulus.

\section{DISCUSSION}

\section{Two components of learning in the VOR}

Our results reveal two components of learning in the VOR that can be distinguished based on the stimuli that produce them and
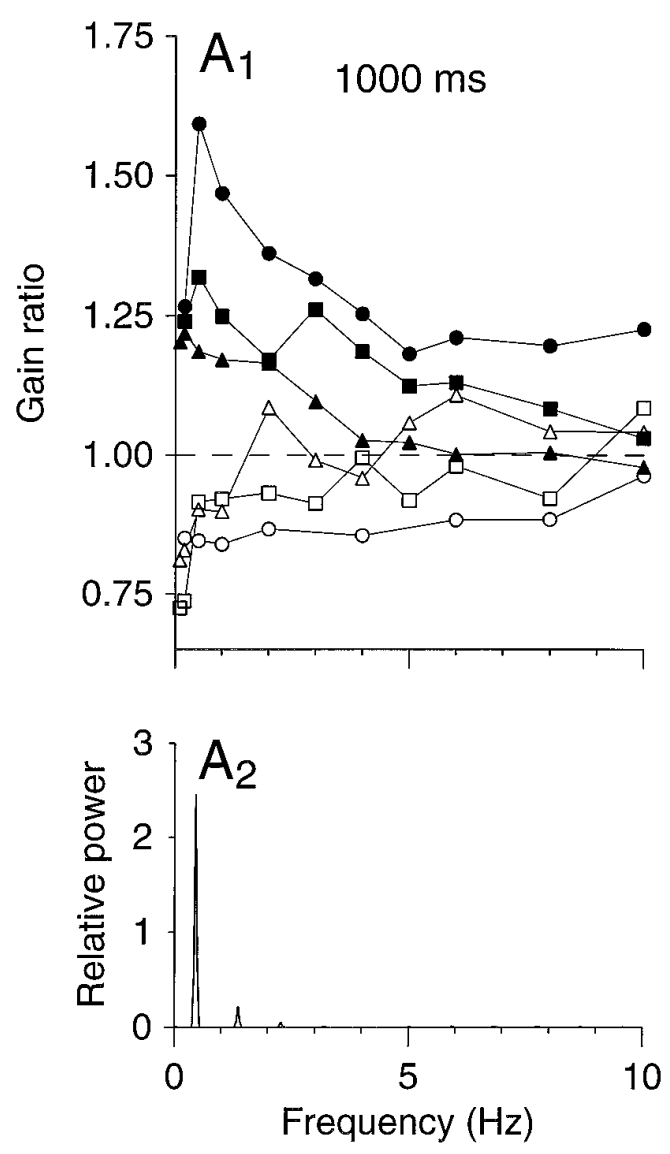
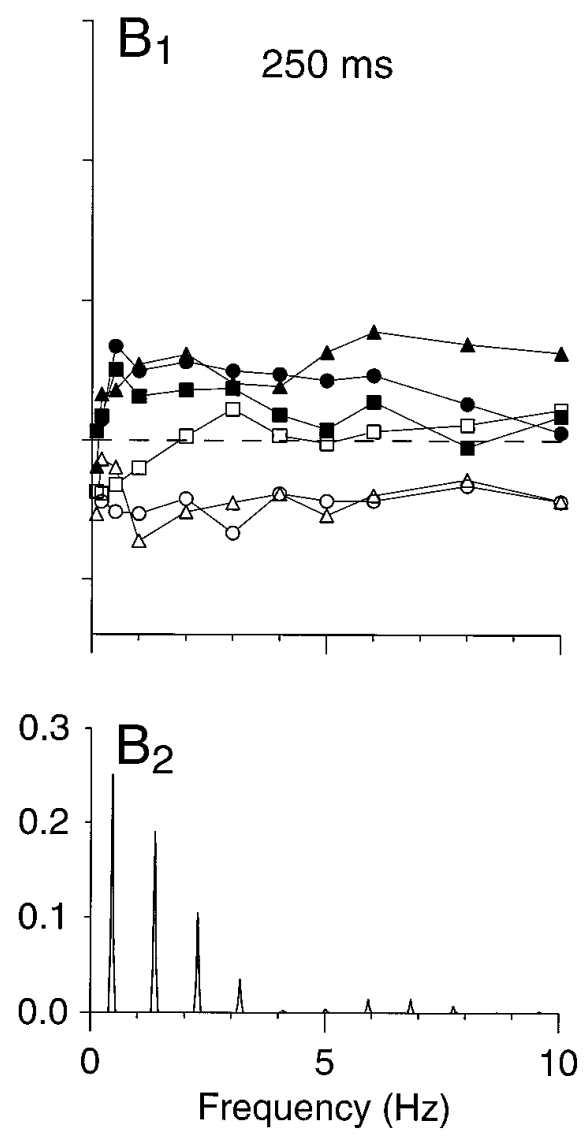

Figure 9. Learned changes in the VOR induced by training with pulse stimuli and tested with sinusoidal vestibular stimuli. $A, 1000$ msec training stimuli; $B$, $250 \mathrm{msec}$ training stimuli. $A 1, B 1$, Gain ratio is plotted as a function of test frequency. Open symbols, $\times 0$ stimulus conditions; filled symbols, $\times 2$ stimulus conditions. Circles, Monkey A; squares, monkey D; triangles, monkey E. A2, B2, Estimated power spectra of the training stimuli. Power is normalized to the power contained in a sinusoidal stimulus with a peak-to-peak amplitude of $20 \%$ sec. Note the different vertical scales in $A 2$ and $B 2$. 
on whether there are changes in just the amplitude or in both the amplitude and dynamics of the VOR. For pulses of head velocity, changes in the dynamics of the VOR are expressed as learned changes that have different amplitudes at different times after the onset of the head turn. For sinusoidal stimuli, the changes in dynamics we focus on are those expressed as "frequencyselective" changes in gain. Minor effects of training on dynamics also can be seen in the small but consistent changes in phase that were observed after training at all frequencies. If one compares the effects of short-duration $(80-250 \mathrm{msec})$ pulse stimuli with the effects of high-frequency $(5 \mathrm{~Hz})$ sinusoidal stimuli, and if one compares the effects of long-duration (500-1000 msec) stimuli with the effects of low-frequency $(0.5 \mathrm{~Hz})$ stimuli, then the analyses in the time and frequency domains lead to consistent conclusions. One component of learning in the VOR is driven by short-duration or high-frequency training stimuli. It produces changes in the amplitude of the VOR but little or no change in its dynamics. Another component of learning is driven by longduration or low-frequency sensory stimuli and produces marked changes in the dynamics as well as the amplitude of the VOR.

The differential effects of different training stimuli on the dynamics of the VOR seem to be attributable to the temporal properties of the individual training stimuli. When the effects of long and short stimuli were compared after training periods that controlled for total duration of visual-vestibular stimulation, long stimuli still produced changes in the dynamics of the reflex, whereas short stimuli did not (Fig. 4B). Furthermore, learning depended on the relative timing of the vestibular and visual stimuli, even when the duration of the vestibular stimulus, visual stimulus, and period of overlap were the same (Figs. 5, 6). Similarly, in the frequency domain, high- and low-frequency sinusoidal training stimuli induced different changes in the dynamics of the VOR after identical training periods (Fig. 7).

The learned changes in dynamics corresponded to greater effects of learning on the low frequency or late components of the VOR than on the high frequency or early components, suggesting that the low frequency or late components of the VOR may be capable of a more extensive repertoire of adaptive processes than the high frequency components. Several previous studies are consistent with this idea. After adaptation with free head movements and magnifying spectacles, Paige and Sargent (1991) reported greater changes in the VOR measured at low frequencies than at high frequencies. Lisberger and Pavelko (1986) reported that adaptation with magnifying and miniaturizing spectacles resulted in changes in the dynamics of the VOR that corresponded to greater modification of the sustained than of the initial phase of VOR. Based principally on the time course of readaptation of the VOR to normal vision after adaptation to dove prisms, Melvill Jones and Gonshor (1982) suggested that the fully adapted, vision-reversed condition comprised two separate components, one a simple gain attenuation that affected all frequencies tested, and a second, reversed-phase component that was present in the low- but not high-frequency VOR responses. Finally, Broussard and Bhatia (1995) found full recovery of the gain of the lowfrequency but not the high-frequency VOR after unilateral peripheral vestibular inactivation.

\section{Possible neural mechanisms for differential regulation of dynamics and gain}

Given what is known about the neural circuitry for the VOR, there are several mechanisms by which the gain and dynamics of the VOR might be differentially regulated. Two sites of plasticity contribute to motor learning in the VOR: there are changes in the vestibular inputs to the cerebellar cortex of the floccular complex, and there are changes in the vestibular inputs to neurons in the vestibular nucleus that are targets of inhibition from the floccular complex (Dufosse et al., 1978; Miles et al., 1980b; Watanabe, 1984; Lisberger and Pavelko, 1988; Lisberger et al., 1994b,c; Luebke and Robinson, 1994; Pastor et al., 1994; Partsalis et al., 1995). It is possible that the two components of learning identified in the present paper correspond directly to the two sites of plasticity that have been proposed, or that learning of gain and dynamics occurs separately at these two sites. Both of these ideas are consistent with the finding that acute cerebellectomy reduced learned changes in the sustained component of the VOR but had no effect on learning expressed in the first $50 \mathrm{msec}$ of the VOR (Pastor et al., 1994). A similar model of separate anatomical sites for storing the learned amplitude and dynamics of a movement has been proposed for classical conditioning of the eyeblink response, another form of cerebellum-dependent learning (Perrett et al., 1993).

The regulation of dynamics could be accomplished at a single anatomical site through the differential modulation of parallel, frequency-selective "channels" at that site (Lisberger et al., 1983). One specific implementation of this model relied on different filtering properties in separate channels of the neural integrator, a mechanism that is rendered plausible by the finding of changes in eccentric gaze holding after modification of the VOR (Tiliket et al., 1994). A previous suggestion from our laboratory was that changes in the dynamics of the VOR after learning might result from separate modifiable and unmodifiable VOR pathways that receive vestibular inputs with different dynamics (Lisberger and Pavelko, 1986). This hypothesis is weakened, however, by studies that suggest that the vestibular afferents contributing to the VOR do not exhibit a wide enough dynamic range to account for the dynamics of the behavior (Lisberger et al., 1983; Minor and Goldberg, 1991; Bronte-Stewart and Lisberger, 1994).

Alternatively, changes in the dynamics of the VOR could be an emergent property of the circuit for the VOR resulting from the feedback loop between the two sites of plasticity. A computational analysis of the circuit for the VOR (Lisberger and Sejnowski, 1992; Lisberger, 1994) suggested that learned changes in gain alone would require parallel changes in the cerebellar cortex and the vestibular nucleus, whereas changes in both gain and dynamics would result when the changes at the two sites were not balanced. If the changes at the two sites are guided by different plasticity mechanisms, then it seems likely that some training stimuli would cause plasticity at one site more than at the other, resulting in unbalanced changes at the two sites and hence a change in the dynamics of the VOR. Stimuli that produced balanced changes at the two sites would alter the gain of the VOR without affecting dynamics.

\section{Implications for the neural signals that guide learning}

We view the behavioral experiments reported here as a step toward identifying the neural signals that guide the cellular mechanisms of plasticity for motor learning in the VOR. The neural pathways that carry visual and vestibular signals to the sites of plasticity for the VOR have dynamics that must certainly transform these signals. Our results place numerous constraints on the transformations that occur in the signals involved in motor learning in the VOR. First, signals involved in at least one component of learning cannot be low-pass-filtered; they must be present in response to stimuli at least as short as $80 \mathrm{msec}$ and must be 
modulated at $5 \mathrm{~Hz}$. Second, signals involved in at least one component of learning cannot be high-pass-filtered; they must be present throughout a constant velocity stimulus for at least $1 \mathrm{sec}$, because progressively bigger changes were produced in the later phase of the VOR by progressively longer stimuli in the range tested. Third, different times in the constant velocity vestibular pulse stimulus must have different representations at the site of plasticity that enable the plasticity mechanism to distinguish between early and late presentation of visual stimuli, and timing information present in the vestibular signals must be able to regulate whether learning involves changes in amplitude alone or changes in the amplitude and dynamics of the VOR. These last conditions are required to account for our observation that image motion paired with the beginning of a vestibular stimulus produced a change in the amplitude of the VOR, but the same image motion presented at later times in the vestibular stimulus produced a change in dynamics as well as amplitude.

Finally, the learning mechanism seems to compensate for a difference in the latency for visual and vestibular signals to arrive at the sites of plasticity. The latencies for vestibular inputs to the vestibular nucleus and cerebellar cortex are $\sim 10-20 \mathrm{msec}$, whereas the latency for visual inputs is close to $100 \mathrm{msec}$ (Baker et al., 1969; Precht and Baker, 1972; Highstein, 1973; Miles et al., 1980a; Lisberger and Pavelko, 1988; Stone and Lisberger, 1990a,b; Lisberger et al., 1994b,c). Thus, visual and vestibular stimuli that are present simultaneously may be represented as nonsimultaneous or time-shifted signals in visual and vestibular inputs at the sites of plasticity. A simple prediction from these arguments would be that the time delay between vestibular and visual signals reaching one or both sites of plasticity would be manifested as a substantial phase shift during training at high sinusoidal frequencies. For example, for $\times 0$ sinusoidal stimuli at low frequencies $(0.1-0.5$ $\mathrm{Hz}$ ), visual climbing fiber inputs are out of phase with inputs from the ipsilateral horizontal canal; conversely, climbing fiber inputs are in phase with inputs from the ipsilateral horizontal canal for $\times 2$ stimuli at low frequencies (Ghelarducci et al., 1975; Watanabe, 1984; Graf et al., 1988; Stone and Lisberger, 1990a,b). At $5 \mathrm{~Hz}$, however, a $100 \mathrm{msec}$ difference in latencies should phaseshift the visual stimulus by $180^{\circ}$ so that visual inputs to the sites of plasticity would be in phase with inputs from the ipsilateral horizontal canal for $\times 0$ stimuli.

If the plasticity mechanisms were coincidence detectors, and $\times 2$ training conditions cause an increase in the gain of the VOR at 0.5 $\mathrm{Hz}$, then a straightforward prediction would be that $\times 0$ stimuli at $5 \mathrm{~Hz}$ also should cause an increase in the gain of the VOR. Alternatively, the neural pathways might filter out visual and/or vestibular signals at high frequencies, avoiding this potential timing problem but resulting in no learning at high frequencies. Our data are not consistent with either of these expectations. Sinusoidal head and target motion at $5 \mathrm{~Hz}$ did cause learning in the VOR, and the learning was in the adaptive direction: increases in the gain of the VOR for $\times 2$ training conditions and decreases for $\times 0$ training conditions. We conclude that at least one component of learning is sensitive to inputs that are modulated at frequencies of at least $5 \mathrm{~Hz}$, and that the signal transformations in the inputs to this component must compensate for the difference in the latencies of the visual and vestibular inputs. One way to accomplish this would be to incorporate a $100 \mathrm{msec}$ delay in the vestibular pathway. Evidence suggests that there is no such delay in the electrical responses in vestibular pathways to the putative sites of plasticity (Baker et al., 1969; Precht and Baker, 1972; Highstein, 1973; Lisberger and Pavelko, 1988; Lisberger et al., 1994b,c). It may be that temporal transformations in the subcellular signaling pathways enable one of the relevant cellular mechanisms of plasticity to compare a visual input with a vestibular input that arrived $100 \mathrm{msec}$ earlier.

\section{REFERENCES}

Baker R, Berthoz A (1975) Is the prepositus hyposglossi nucleus the source of another vestibulo-ocular pathway? Brain Res 86:121-127.

Baker R, Mano N, Shimazu H (1969) Postsynaptic potentials in abducens motoneurons induced by vestibular stimulation. Brain Res 15:577-580.

Baker RG, Precht W, Llinas R (1972) Cerebellar modulatory action on the vestibulo-trochlear pathway in the cat. Exp Brain Res $15: 364-385$.

Bronte-Stewart HM, Lisberger SG (1994) Physiological properties of vestibular afferents that mediate motor learning and normal performance of the vestibulo-ocular reflex in monkeys. J Neurosci $6: 346-354$

Broussard DM, Bhatia JK (1995) A comparison of optically-induced motor learning and compensation for unilateral damage by the VOR. Soc Neurosci Abstr 21:139.

Collewijn H, Grootendorst AF (1979) Adaptation of the optokinetic and vestibulo-ocular reflexes to modified visual input in the rabbit. Prog Brain Res 50:771-781.

Dufosse M, Ito M, Jastreboff PJ, Miyashita Y (1978) A neural correlate in rabbit's cerebellum to adaptive modification of the vestibulo-ocular reflex. Brain Res 150:611-616.

Gauthier GM, Robinson DA (1975) Adaptation of the human vestibuloocular reflex to magnifying lenses. Brain Res 92:331-335.

Ghelarducci B, Ito M, Yagi N (1975) Impulse discharges from flocculus Purkinje cells of alert rabbits during visual stimulation combined with horizontal head rotation. Brain Res 87:66-72.

Godaux E, Halleux J, Gobert C (1983) Adaptive change of the vestibuloocular reflex in the cat: the effects of a long-term frequency-selective procedure. Exp Brain Res 49:28-34.

Gonshor A, Melvill Jones G (1973) Changes of human vestibulo-ocular response induced by vision-reversal during head rotation. J Physiol (Lond) 234:102P-103P.

Graf W, Simpson JI, Leonard CS (1988) Spatial organization of visual messages of the rabbit's cerebellar flocculus. II. Complex and simple spike responses of Purkinje cells. J Neurophysiol 60:2091-2121.

Highstein SM (1973) Synaptic linkage in the vestibulo-ocular and cerebello-vestibular pathways to the VIth nucleus in the rabbit. Exp Brain Res 17:301-314.

Ito, M, Shiida T, Yagi N, Yamamoto M (1974) The cerebellar modification of rabbit's horizontal vestibulo-ocular reflex induced by sustained head rotation combined with visual stimulation. Proc Jpn Acad 50:85-89.

Ito M, Nisimaru N, Yamamoto M (1976) Pathways for the vestibuloocular reflex excitation arising from semicircular canals of rabbits. Exp Brain Res 24:257-271.

Ito M, Nisimaru N, Yamamoto M (1977) Specific patterns of neuronal connexions involved in the control of the rabbit's vestibulo-ocular reflexes by the cerebellar flocculus. J Physiol (Lond) 265:833-854.

Keller EL (1978) Gain of the vestibulo-ocular reflex in monkey at high rotational frequencies. Vision Res 18:311-315.

Langer T, Fuchs AF, Scudder CA, Chubb MC (1985) Afferents to the flocculus of the cerebellum in the rhesus macaque as revealed by retrograde transport of horseradish peroxidase. J Comp Neurol 235:1-25.

Lisberger SG (1994) Neural basis for motor learning in the vestibuloocular reflex of primates. III. Computational and behavioral analysis of the sites of learning. J Neurophysiol 72:974-998.

Lisberger SG, Pavelko TA (1986) Vestibular signals carried by pathways subserving plasticity of the vestibulo-ocular reflex in monkey. J Neurosci 6:346-354.

Lisberger SG, Pavelko TA (1988) Brain stem neurons in modified pathways for motor learning in the primate vestibulo-ocular reflex. Science 242:771-773.

Lisberger SG, Sejnowski TJ (1992) Motor learning in a recurrent network model based on the vestibulo-ocular reflex. Nature 360:159-161.

Lisberger SG, Miles FA, Optican LM (1983) Frequency-selective adaptation: evidence for channels in the vestibulo-ocular reflex? J Neurosci 3:1234-1244. 
Lisberger SG, Pavelko TA, Broussard DM (1994a) Responses during eye movements of brain stem neurons that receive monosynaptic inhibition from the flocculus and ventral paraflocculus in monkeys. J Neurophysiol 72:909-927.

Lisberger SG, Pavelko TA, Broussard DM (1994b) Neural basis for motor learning in the vestibuloocular reflex of primates. I. Changes in the responses of brain stem neurons. J Neurophysiol 72:928-953.

Lisberger SG, Pavelko TA, Bronte-Stewart HM, Stone LS(1994c) Neural basis for motor learning in the vestibuloocular reflex of primates. II. Changes in the responses of horizontal gaze velocity Purkinje cells in the cerebellar flocculus and ventral paraflocculus. J Neurophysiol 72:954-973.

Luebke AE, Robinson DA (1994) Gain changes of the cat's vestibuloocular reflex after flocculus deactivation. Exp Brain Res 98:379-390.

Melvill Jones G, Gonshor A (1982) Oculomotor response to rapid head oscillation $(0.5-5.0 \mathrm{~Hz})$ after prolonged adaptation to vision-reversal: "simple" and "complex" effects. Exp Brain Res 45:45-58.

Miles FA, Fuller JH (1974) Adaptive plasticity in the vestibulo-ocular responses of the rhesus monkey. Brain Res 80:512-516.

Miles FA, Fuller JH, Braitman DJ, Dow BM (1980a) Long-term adaptive changes in primate vestibuloocular reflex. III. Electrophysiological observations in flocculus of normal monkeys. J Neurophysiol 43:1437-1476.

Miles FA, Braitman DJ, Dow BM (1980b) Long-term adaptive changes in primate vestibuloocular reflex. IV. Electrophysiological observations in flocculus of adapted monkeys. J Neurophysiol 43:1477-1493.

Minor LB, Goldberg JM (1991) Vestibular-nerve inputs to the vestibuloocular reflex: a functional ablation study in the squirrel monkey. J Neurosci 11:1636-1648.

Paige GD, Sargent EW (1991) Visually-induced adaptive plasticity in the human vestibulo-ocular reflex. Exp Brain Res 84:25-34.

Partsalis AM, Zhang Y, Highstein SM (1995) Dorsal Y group in the squirrel monkey. II. Contribution of the cerebellar flocculus to neuronal responses in normal and adapted animals. J Neurophysiol 73:632-649.
Pastor AM, de la Cruz RR, Baker R (1994) Cerebellar role in adaptation of goldfish vestibuloocular reflex. J Neurophysiol 72:1383-1394.

Perrett SP, Ruiz BP, Mauk MD (1993) Cerebellar cortex lesions disrupt learning-dependent timing of conditioned eyelid responses. J Neurosci 13:1708-1718.

Powell KD, Quinn KJ, Rude SA, Peterson BW, Baker JF (1991) Frequency dependence of cat vestibulo-ocular reflex direction adaptation: single frequency and multifrequency rotations. Brain Res 550:137-141.

Precht W, Baker R (1972) Synaptic organization of the vestibulotrochlear pathway. Exp Brain Res 15:158-184.

Precht W, Llinas R (1969) Functional organization of the vestibular afferents to the cerebellar cortex of frog and cat. Exp Brain Res 9:30-52

Shimazu H, Precht W (1966) Inhibition of central vestibular neurons from the contralateral labyrinth and its mediating pathway. J Neurophysiol 29:467-492.

Simpson JI, Alley KE (1974) Visual climbing fiber input to rabbit vestibulo-cerebellum: a source of direction-specific information. Brain Res 82:302-308.

Stone LS, Lisberger SG (1990a) Visual responses of Purkinje cells in the cerebellar flocculus during smooth pursuit eye movements in monkeys. I. Simple spikes. J Neurophysiol 63:1241-1261.

Stone LS, Lisberger SG (1990b) Visual responses of Purkinje cells in the cerebellar flocculus during smooth pursuit eye movements in monkeys. II. Complex spikes. J Neurophysiol 63:1262-1275.

Tiliket C, Shelhamer M, Roberts D, Zee DS (1994) Short-term vestibuloocular reflex adaptation in humans. I. Effect on the ocular motor velocity-to-position neural integrator. Exp Brain Res 100:316-327.

Watanabe E (1984) Neuronal events correlated with long-term adaptation of the horizontal vestibulo-ocular reflex in the primate flocculus. Brain Res 297:169-174.

Wurtz RH (1969) Visual receptive fields of striate cortex neurons in awake monkeys. Neurosci Res 32:727-742. 